\section{Block-Coded PSK Modulation Using Two-Level Group Codes Over Dihedral Groups}

Jyoti Bali, Student Member, IEEE, and B. Sundar Rajan, Member, IEEE

\begin{abstract}
A length $n$ group code over a group $G$ is a subgroup of $G^{n}$ under component-wise group operation. Group codes over dihedral groups $D_{M}$, with $2 M$ elements, that are two-level constructible using a binary code and a code over $Z_{M}$ residue class integer ring modulo $M$, as component codes are studied for arbitrary $M$. A set of necessary and sufficient conditions on the component codes for the two-level construction to result in a group code over $D_{M}$ are obtained. The conditions differ for $M$ odd and even. Using two-level group codes over $D_{M}$ as label codes, performance of block-coded modulation scheme is discussed under al possible matched labelings of $2 M$-APSK and $2 M$-SPSK (asymmetric and symmetric PSK) signal sets in terms of the minimum squared Euclidean distance. Matched labelings that lead to Automorphic Euclidean Distance Equivalent codes are identified. It is shown that depending upon the ratio of Hamming distances of the component codes some labelings perform better than other. The best labeling is identified under a set of restrictive conditions. Finally, conditions on the component codes for phase rotational invariance properties of the signal space codes are discussed.
\end{abstract}

Index Terms-Coded modulation, dihedral groups, group codes, multilevel codes.

\section{INTRODUCTION}

A multilevel block code [1]-[10] of $L$ levels uses $L$ block codes each of the same length $n$, called component codes, over finite alphabets of possibly different sizes. A signal set $S$, called the basic signal set, of dimension $N$, has $\prod_{i=1}^{L} m_{i}$ points, where $m_{i}$, $i=1,2, \cdots, L$ are the size of the alphabets, with each point labeled by an ordered $L$-tuple with one entry from each alphabet. With this labeling, a set of $L$ codewords, one from each code, correspond to

Manuscript received May 20, 1997. This work was supported by the Council of Scientific and Industrial Research (CSIR), India, through a fellowship 9/86(320)/95-EMR-I to J. Bali. The material in this correspondence was presented in part at the IEEE Information Theory Symposium, Ulm, Germany, June 20-July 4, 1997. Part of this correspondence appears in Abstracts: Mediterranean Workshop on Coding and Information Integrity (Palma, Spain, February 28-March 1, 1997), and a part of Section IV in Proceedings of ISIT '97 (Ulm, Germany).

J. Bali is with the Department of Electrical Engineering, Indian Institute of Technology, Delhi, Hauz Khas, New Delhi, 110016, India (e-mail: jbali@ee.iitd.ernet.in).

B. Sundar Rajan is with the Electrical Communication Engineering Department, Indian Institute of Science, Bangalore, 560 012, India (e-mail: bsrajan@ece.iisc.ernet.in).

Publisher Item Identifier S 0018-9448(98)03459-2. a point in $N n$ dimensions, with each coordinate of $L$ codewords choosing a point in $S$. Multilevel coded signal sets with linear codes over GF (2) as component codes have been studied in [1]-[5] and in various general settings in [6]-[10]. Kschischang et al. [11] use linear codes over nonbinary fields to construct multilevel signal sets and give algebraic structural properties of these codes. Multilevel codes for the purpose of unequal error protection have been discussed in [12] and [13]. Suboptimal multistage decoding and performance analysis of multilevel codes have been studied in [14]-[16].

This correspondence deals with two-level $(L=2)$ group codes with the basic signal set consisting of points on a circle. The block diagram of a two-level block-coded modulation is shown in Fig. 1(a). When $C_{s}$ and $C_{r}$ are length $n$ codes over alphabets $Y=\left\{y_{1}, y_{2}\right\}$ $\left(m_{1}=2\right)$ and $X=\left\{x_{1}, x_{2}, x_{3}, x_{4}\right\}\left\{m_{2}=4\right\}$, Fig. 1(b) shows a labeling of $S$ consisting of eight points on the circle with $X$ and $Y$. For codewords $a=\left(a_{0}, a_{1}, \cdots, a_{n-1}\right) \in C_{s}$ and $b=\left(b_{0}, b_{1}, \cdots, b_{n-1}\right) \in C_{r}$, each pair $\left(a_{i}, b_{i}\right), i=0,1, \cdots, n-1$, selects a point in $S$, and the pair $(a, b)$ specify a point in $2 n$ dimensions. The collection of all such points in $2 n$ dimensions corresponding to all possible pairs of codewords constitute the twolevel block-coded modulation code (signal set) or signal space code. This correspondence concerns $Y$ and $X$ being $Z_{2}$ and $Z_{M}$, residue class integers modulo 2 and $M$, respectively, and the basic signal set being a collection of $2 M$ points on a unit circle matched to the dihedral group with $2 M$ elements.

A signal set $S$ is said to be matched to a group $G$ if there exists a mapping $\mu$ from $G$ onto $S$ such that for all $g$ and $g^{\prime}$ in $G$

$$
d_{E}\left(\mu(g), \mu\left(g^{\prime}\right)\right)=d_{E}\left(\mu\left(g^{-1} g^{\prime}\right), \mu(e)\right)
$$

where $d_{E}(a, b)$ denotes the squared Euclidean distance between $a$, $b \in S$, and $e$ is the identity element of $G$. If $G$ and $S$ have the same number of elements then the elements of $S$ can be labeled with the elements of $G$, and such a labeling satisfying condition (1) is referred to as a matched labeling [17], [18]. A signal set matched to a group has the property that the Euclidean distance distribution of the points of the signal set from any point is the same.

A group code over a group $G$ is a subgroup $C$ of $G^{n}$ with componentwise group operation. Most known good classes of signal space codes are geometrically uniform codes [19] for which group codes constitute a basic ingredient. If $S$ is a signal set of dimension $N$ matched to a group $G$ and $\mu$ is a matched labeling, then under the extended mapping

$$
\begin{aligned}
\mu^{n}: G^{n} \rightarrow S^{n} \text { given by } \mu^{n}\left(g_{0}, g_{1}, \cdots, g_{n-1}\right) & \\
& =\left(\mu\left(g_{0}\right), \mu\left(g_{1}\right), \cdots, \mu\left(g_{n-1}\right)\right)
\end{aligned}
$$

$\mu^{n}(C)$ gives a signal set in $N n$ dimensions, called the signal space code, and this is matched to the group $C$, and hence is geometrically uniform [19]. $C$ is referred to as the label code of the signal space code. The squared Euclidean distance distribution determines the performance of the signal set when used in an additive white Gaussian noise channel, and generally the minimum squared Euclidean distance (MSED) is taken to be the performance index. Given a group $G$ and a signal set $S$, the matching labeling of $S$ with $G$ is not unique. So, given a group code the resulting signal space code depends on the particular matched labeling used, and hence the performance is dependent on the choice of the matched labeling. The choice of the best labeling for a label code then becomes an important problem.

In [1], Biglieri and Caire have studied the geometrical uniformity properties of signal space codes generated by multilevel construction. 


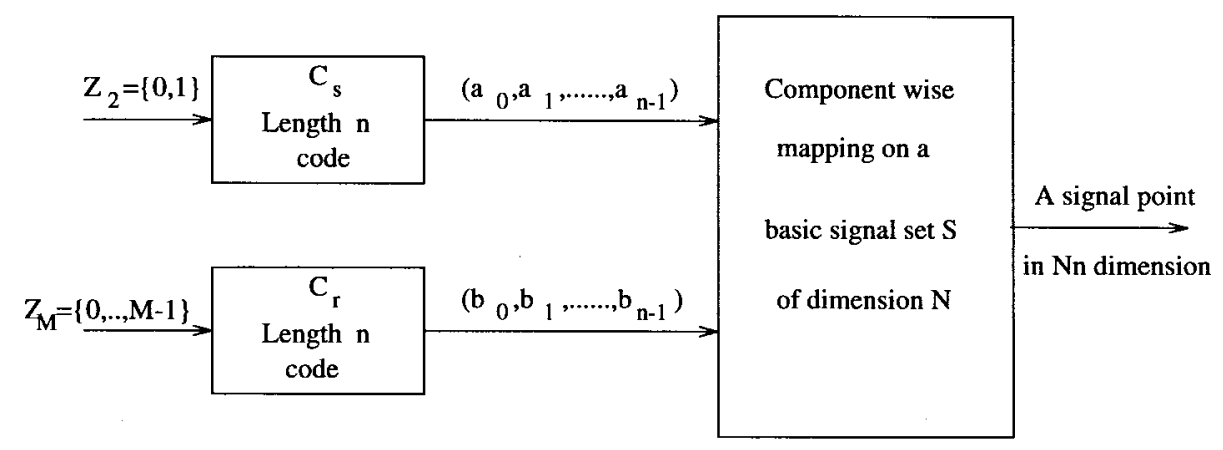

(a)

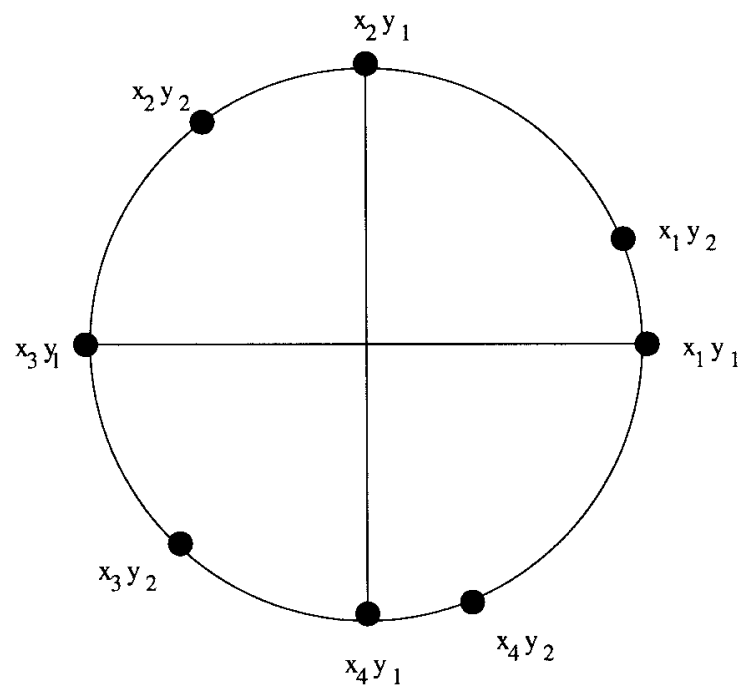

(b)

Fig. 1. (a) Block diagram of a two-level block-codes modulation. (b) Labeling of an 8-PSK signal set with $X$ and $Y$.

The component codes used are $L$ linear binary codes with the $2^{L}$ ary SPSK as the basic signal set. The points of PSK signal set can be designated with either the cyclic group with $2^{L}$ elements or with the dihedral group with $2^{L}$ elements. They derive conditions under which the resulting multilevel code is a group code over cyclic or dihedral group. We study in this correspondence two-level group codes over dihedral groups with $2 M$ elements, denoted by $D_{M}$, with one component code a binary code and the other a code over $Z_{M}$. To be specific, the component codes are i) $C_{s}$, a code over $Z_{2}=\{0,1\}$ and ii) $C_{r}$, a code over $Z_{M}=\{0,1, \cdots, M-1\}$. The codes of [1] and [3] are special cases corresponding to $M=2^{L-1}$ and $C_{r}$ being a decomposable code [3]. There are several ways of achieving matched labeling of a $2 M$-PSK signal set with $D_{M}$. In Section II, we describe all possible matched labelings (Definition 1) of the $2 M$-PSK signal set, both symmetric ( $2 M$-SPSK) and asymmetric ( $2 M$-APSK) with $D_{M}$. Generally, the labeling that is studied is one that maps $s \in D_{M}$ to a point closest to the image of the identity element, which we refer to as the Standard Labeling $(S L)$. The labeling that maps $s$ to a signal point that is farthest away from the image of the identity element is referred to as Maximum Distance Labeling $(M D L)$. Also the labelings that lead to automorphic Euclidean distance equivalent [20] signal space codes are identified. In Section III we first obtain conditions (Theorem 1) on the component codes under which the resulting two-level code is a group code over $D_{M}$ and relate these conditions to those obtained in [1] and [7]. Section IV contains the main results of this correspondence: Euclidean distance properties of signal space codes obtained from two-level group codes over $D_{M}$ as label codes with $2 M$-SPSK as well as $2 M$ -
APSK signal set as the basic signal set are studied under general labelings. Conditions under which certain labelings give larger MSED compared to a class of other matched labelings are derived. It turns out that the ratio of Hamming distances of the component codes $C_{s}$ and $C_{r}$ can be used to obtain several Euclidean distance properties of the signal space code. For a case, with rather restrictive conditions, the best matched labeling is identified. 6-SPSK- and 4APSK-based modulation schemes are discussed as special cases. The phase rotational properties are discussed in Section V. Finally, some concluding remarks including possible directions for further research are given in Section VI.

\section{Labeling of PSK Signals Set with Dihedral Groups}

Let $r$ and $s$ be the generators of the dihedral group with $2 M$ elements

$D_{M}=\left\{r^{i} s^{j} \mid r^{M}=e, s^{2}=e, r^{i} s=s r^{-i}, 0 \leq i<M, j=0,1\right\}$

where $e$ is the identity of $D_{M}$. The group operation can be expressed as

$$
\left(r^{i_{1}} s^{j_{1}}\right)\left(r^{i_{2}} s^{j_{2}}\right)=r^{i_{1}+i_{2}\left(1-2 j_{1}\right)} s^{j_{1}+j_{2}}
$$

and the inverse of an element is given by $\left(r^{i} s^{j}\right)^{-1}=r^{i(2 j-1)} s^{j}$. Let $\mu$ be a matched labeling of a $2 M$-PSK signal set, i.e.,

$$
\begin{aligned}
d_{E}\left(\mu\left(r^{i_{1}} s^{j_{1}}\right), \mu\left(r^{i_{2}}, s^{j_{2}}\right)\right)= & d_{E}\left(\mu\left(\left(r^{i_{1}} s^{j_{1}}\right)^{-1}\left(r^{i_{2}} s^{j_{2}}\right)\right), \mu(e)\right), \\
& 0 \leq i_{1}, i_{2}<M, \quad j_{1}, j_{2}=0,1 .
\end{aligned}
$$




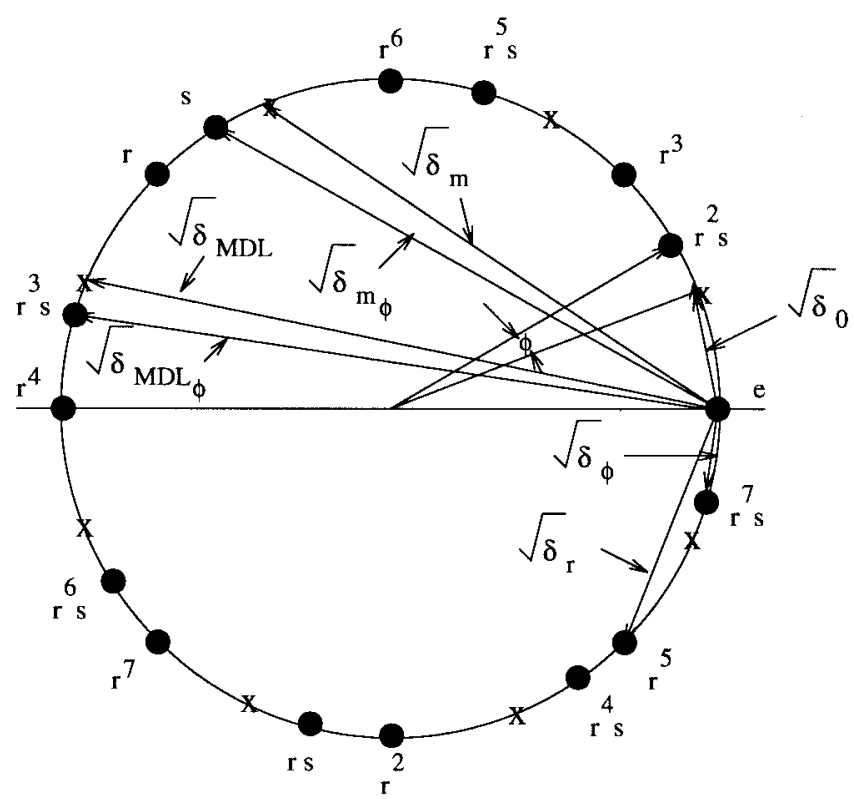

(a)

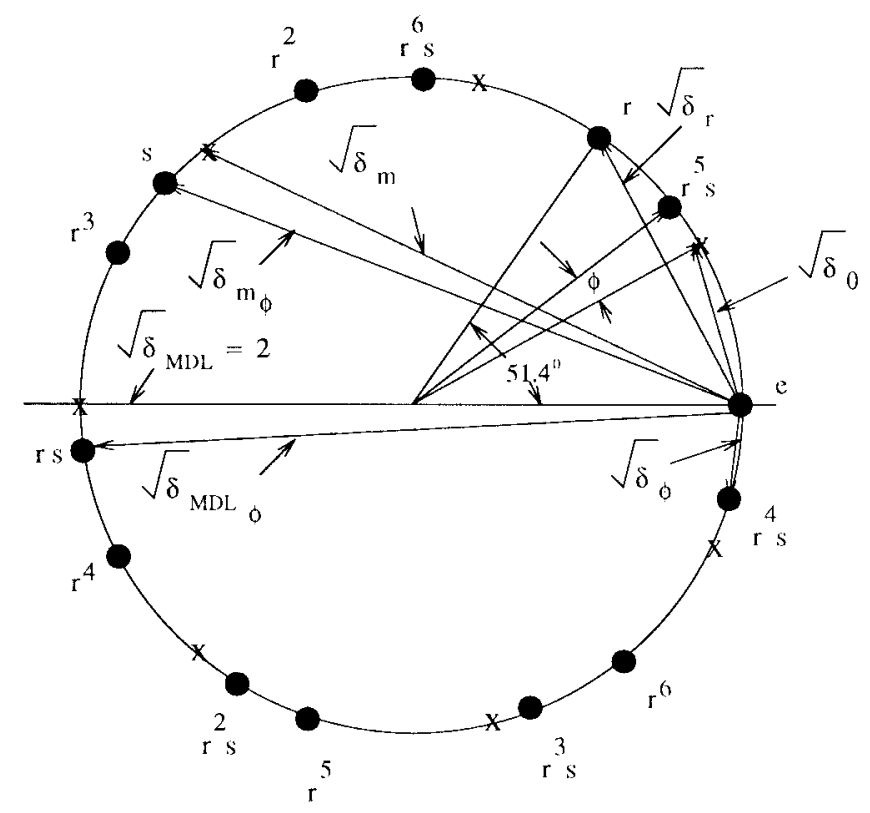

(b)

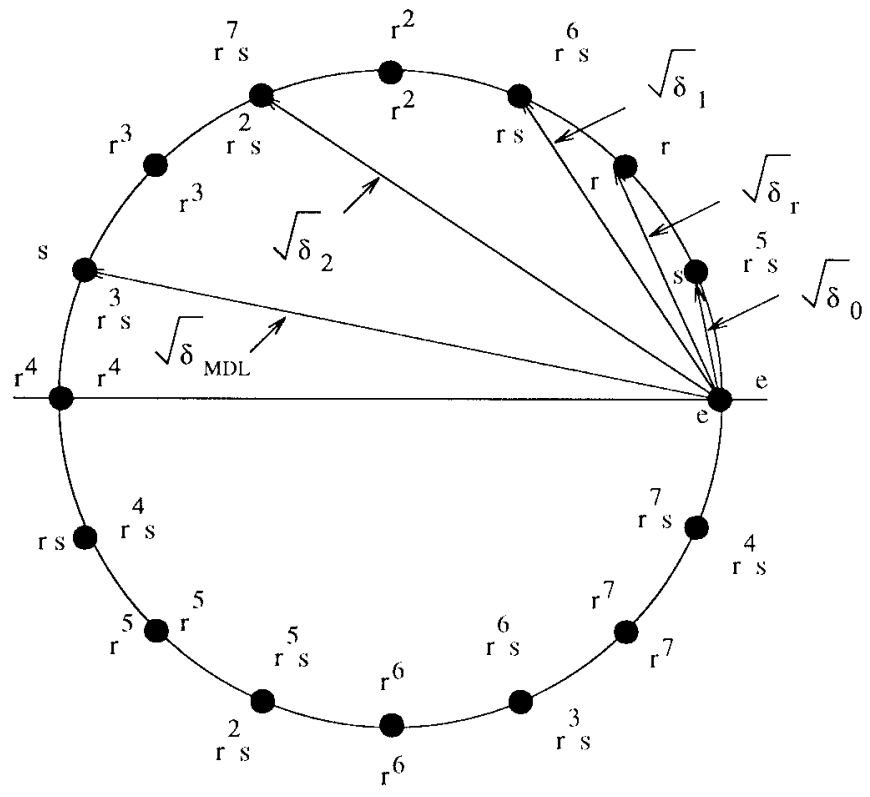

(c)

Fig. 2. (a) Matched labeling of a 16-APSK signal set corresponding to $M=8, \phi \neq 0,1=3, m=2$ in (4). (The points marked $x$ adjacent to all the points marked $r^{3} s, j=0,1, \cdots, 7$, are those to which $r^{3}$ 's will be mapped if $\phi=0$, i.e., for the 16-SPSK.) (b) Matched labeling of a 14-APSK signal set for $M=7, \phi \neq 0, m=2$ in (5). (The points marked $x$ adjacent to all the points marked $r^{j}$ 's, $j=0,1, \cdots, 6$, are those to which $r^{j}$ 's will be mapped if $\phi=0$, i.e., for the 14-SPSK.) (c) Standard labeling (SL) (inner) and maximum-distance labeling ( $M D L$ ) (outer) of a 16-APSK signal set as per (5).

Throughout, it is assumed that $e$ is mapped onto the point $(1,0)$ on the unit circle. The signal points are $e^{\sqrt{-1} \phi}, 0 \leq \phi<2 \pi$. For convenience, hereafter, $\mu(\cdot)=e^{\sqrt{-1} \phi}$ will be written only as $\mu(\cdot)=\phi$.

Definition 1: A matched labeling $\mu$ of a $2 M$-APSK signal set matched to $D_{M}$ is said to be an $m$-labeling, $0 \leq m \leq M-1$, with angle of asymmetry $\phi,-\pi / 2 M<\phi<\pi / 2 M$, and denoted by $m L_{\phi}$, if

$$
\begin{aligned}
\mu\left(r^{i} s^{j}\right)=j((2 m+1) \pi / M+\phi) & +i l(2 \pi / M), \\
i & =0,1, \cdots, M-1, \quad j=0,1
\end{aligned}
$$

where $(l, M)=1$, i.e., $l$ and $M$ are relatively prime. The matched labeling $\mu$ is said to be maximum-distance labeling $\left(M D L_{\phi}\right)$ if
$m=M / 2-1$ (for $M$ even), or $m=(M-1) / 2$ (for $M$ odd), and standard labeling $\left(S L_{\phi}\right)$ if $m=0$ for $\phi \leq 0$ and $m=M-1$ for $\phi>0$.

That (4) is a matched labeling for all values of $l, M$, and $\phi$ can easily be verified. Labeling of a $2 M$-SPSK is obtained with $\phi=0$, in which case the suffix $\phi$ is dropped in the notations $m L_{\phi}, M D L_{\phi}$, and $S L_{\phi}$. Fig. 2(a) shows a matched labeling corresponding to $M=8$, $l=3, m=2, \phi \neq 0$, and Fig. 2(b) shows a matched labeling corresponding to $M=7, l=1, m=2, \phi \neq 0$. (The distances marked in Fig. 2(a)-(c) are defined in Section IV.) The matched labelings $m L_{\phi}, M D L_{\phi}$, and $S L_{\phi}$ will be collectively referred to as asymmetric labelings and $m L, M D L$, and $S L$ by symmetric labelings.

For $m L_{\phi}$, the image of $s$ is $(2 m+1) \pi / M+\phi$ and the image of $r$ is $l(2 \pi / M)$ which is independent of $m$. The matched labeling 
$\mu$ is completely determined by the images $\mu(s)$ and $\mu(r)$ of the generators of $D_{M}$. In (4), $r$ is mapped onto $e^{\sqrt{-1 l}(2 \pi) / M}$. Without loss of generality, it can be mapped onto $e^{\sqrt{-1}(2 \pi) / M}$, in which case (4) becomes

$$
\begin{aligned}
\mu\left(r^{i} s^{j}\right)=j((2 m+1) \pi / M+\phi)+i(2 \pi / M), & \\
& i=0,1, \cdots, M-1, \quad j=0,1 .
\end{aligned}
$$

There is no loss of generality in (5), since as far as two-level group codes are concerned the signal space codes obtained by labeling as in (4) will lead to signal space codes that are Euclidean distance equivalent to those obtained by the labeling given by (5), i.e., the Euclidean distance distribution of the two signal space codes will be identical (shown in the following subsection). So, throughout we use the labeling given by (5). Fig. 2(c) shows $S L$ and $M D L$ as per (5), of a 16-APSK signal set.

Definition 2: Let $C_{s}$ and $C_{r}$ be length $n$ codes over, respectively, $Z_{2}=\{0,1\}$ and $Z_{M}=\{0,1,2, \cdots, M-1\}$, and

$$
\boldsymbol{a}=\left(a_{0}, a_{1}, \cdots, a_{n-1}\right) \in C_{s}
$$

and

$$
\boldsymbol{b}=\left(b_{0}, b_{1}, \cdots, b_{n-1}\right) \in C_{r} .
$$

Let $\boldsymbol{c}_{\boldsymbol{a}, \boldsymbol{b}}$ denote

$$
\left(r^{b_{0}} s^{a_{0}}, r^{b_{1}} s^{a_{1}}, \cdots, r^{b_{n-1}} s^{a_{n-1}}\right) \in D_{M}^{n} .
$$

The subset $\left\{\boldsymbol{c}_{\boldsymbol{a}, \boldsymbol{b}} \mid \boldsymbol{a} \in C_{s}, \boldsymbol{b} \in C_{r}\right\}$ of $D_{M}^{n}$, denoted by $r^{C_{r}}{ }^{C_{s}}$, is called the two-level code over $D_{M}$ with component codes $C_{s}$ and $C_{r}$. If $r^{C_{r}} s^{C_{s}}$ is a subgroup of $D_{M}^{n}$ then it is called a two-level group code and it is denoted by $C$. The signal space code obtained with $\boldsymbol{C}$ as the label code and $\mu$ as the matched labeling will be denoted by $\mu^{n}(\boldsymbol{C})$, and the signal point in $\mu^{n}(\boldsymbol{C})$ corresponding to $\boldsymbol{a}$ and $\boldsymbol{b}$ by $\mu^{n}(\boldsymbol{a}, \boldsymbol{b})$.

The image of $s$ under a matched labeling uniquely determines the labeling and two matched labelings will lead to signal space codes that differ in performance for the same label code. So, the central problem is choosing the best labeling for a specified label code. This is similar to the initial vector problem for Slepian signal sets, where the signal points are generated by a group of orthogonal matrices isomorphic to the group acting on a vector called initial vector whose choice determines the performance of the signal set [18], [21]. Different values for $\mu(s)$ can also be seen as different coset representative selection when the two-level code is seen as block coset code [11], where each component of a codeword of the binary code $C_{s}$ selects either the normal subgroup $\left\{1, r, r^{2}, \cdots, r^{M-1}\right\}$ of $D_{M}$ or its coset $\left\{s, r s, r^{2} s, \cdots, r^{M-1} s\right\}$, and then the corresponding component of a codeword of the $Z_{M}$ code selects an element from within the subgroup or the coset.

\section{A. Automorphic Euclidean Distance Equivalent Labelings}

Caire and Biglieri [20] introduced the notion of automorphic Euclidean distance equivalent (AEDE) codes: Let $S$ be a signal set matched to a group $G$ and $\mu: G \rightarrow S$, a matched labeling. Two signal space codes $C$ and $C^{\prime}$ over $G$ are called AEDE if there exists an automorphism $f$ of the group $G^{n}$ which maps $C$ to $C^{\prime}$ such that the composition map $\left(\mu^{n} f\left(\mu^{n}\right)^{-1}\right)$ is a symmetry of $S^{n}$.

Definition 3: Two labelings $\mu$ and $\mu^{\prime}$ given by (4) are said to be automorphic Euclidean distance equivalent labelings if $\mu^{n}(C)$ and $\mu^{\prime n}(C)$ are AEDE codes.

Now we show that the labelings given by (4) and (5) are AEDE. Since we shall be concerned with only group codes, it is sufficient to consider the squared Euclidean distance distribution from $\mu^{(n)}(e, e, \cdots, e)$, i.e., the Euclidean weight distribution of the signal space codes. The signal point corresponding to the component codewords

and

$$
\boldsymbol{a}=\left(a_{0}, a_{1}, \cdots, a_{n-1}\right) \in C_{s}
$$

$$
\boldsymbol{b}=\left(b_{0}, b_{1}, \cdots, b_{n-1}\right) \in C_{r}
$$

is $\mu^{n}(\boldsymbol{a}, \boldsymbol{b})$ where $\mu$ is given by (5) and let $\mu^{\prime n}(\boldsymbol{a}, \boldsymbol{b})$ denote the signal point obtained with the matched labeling given by (4). Since $l$ is a unit in $Z_{M}$ and $C_{r}$ is a linear code over $Z_{M}$, there exists a codeword

$$
\begin{aligned}
\gamma=\left(\gamma_{0}, \gamma_{1}, \cdots,\right. & \left.\gamma_{n-1}\right) \in C_{r}, \\
& \text { where } \gamma_{i}=b_{i} l, i=0,1, \cdots, n-1 .
\end{aligned}
$$

Now $\mu^{n}(\boldsymbol{a}, \boldsymbol{\gamma})$ is a signal point which has the same Euclidean weight as that of $\mu^{\prime n}(\boldsymbol{a}, \boldsymbol{b})$. Multiplication by $l$ keeps invariant the code $C_{r}$, and indeed an automorphism of $Z_{M}^{n}$ as well. Under appropriate choice of labelings these two codes over $D_{M}$ lead to signal space codes that have the same Euclidean distance distribution and hence the labeling (5) and (4) are AEDE.

Furthermore, among the labelings given by (5) it is sufficient to consider $m$ in the range $0 \leq m \leq M / 2-1$ for even values of $M$ and $0 \leq m \leq(M-1) / 2$ for odd values of $M$. (In other words, there is no loss of generality in restricting the image of $s$ only to the upper half of the circle.) For the labelings $m L_{\phi}$ and $(M-m-1) L_{-\phi}$ are AEDE, since $\mu^{n}(\boldsymbol{a}, \boldsymbol{b})$ where $\mu$ is $(M-m-1) L_{-\phi}$, has the same Euclidean weight as that of $\mu^{n}(\boldsymbol{a},-\boldsymbol{b})$ where $\mu$ is given by (5), which can be easily verified. Moreover, the map

$$
\left(b_{0}, b_{1}, \cdots, b_{n-1}\right) \rightarrow\left(-b_{0},-b_{1}, \cdots,-b_{n-1}\right)
$$

is an automorphism of the label code. Hence the labelings $m L_{\phi}$ and $(M-m-1) L_{-\phi}$ are AEDE.

\section{Characterization of Two-Level Group Codes over $D_{M}$}

In this section we first give the necessary and sufficient conditions on the component codes of the two-level construction shown in Fig. 1 to result in a group code over $D_{M}$. Then using the fact that dihedral groups are obtainable as semidirect product of its subgroups and the algebraic characterization of multilevel group codes over semidirect product groups given by Garello and Benedetto [7], rederive these conditions.

Theorem 1: The two-level code $C=r^{C_{r}}{ }^{C_{s}}$ is a group code over $D_{M}$ if and only if

i) $C_{s}$ and $C_{r}$ are linear codes over $Z_{2}$ and $Z_{M}$, respectively (9)

ii) $C_{s} \odot 2 C_{r} \subseteq C_{r}$, if $M$ is even and $C_{s} \odot C_{r} \subseteq C_{r}$, if $M$ is odd,

where $\odot$ denotes pointwise product.

Proof: Let $C$ be a group code over $D_{M}$. Then

$$
\left(r^{0} s^{0}, \cdots, r^{0} s^{0}, \cdots, r^{0} s^{0}\right)=(e, e, \cdots, e) \in C
$$

and hence the "all-zero vector" is a codeword in both $C_{r}$ and $C_{s}$. Consider the subcode $r^{C_{r}}$ of $C$, which consists of all codewords of $C$ with $a_{0}=a_{1}=\cdots=a_{n-1}=0$ in Definition 2. Clearly, $r^{C_{r}}$ is a group code over the cyclic group of order $M$. Since every group code over a cyclic group of order $M$ is a linear code over the integer residue ring $Z_{M}$ [22], it follows that $C_{r}$ is a linear code over $Z_{M}$. By similar arguments, $C_{s}$ is a linear code over $Z_{2}$. To show the second condition (10), let

$$
\begin{aligned}
c_{\boldsymbol{a}, \boldsymbol{b}} & =\left(r^{b_{0}} s^{a_{0}}, \cdots, r^{b_{i}} s^{a_{i}}, \cdots, r^{b_{n-1}} s^{a_{n-1}}\right) \\
c_{\boldsymbol{x}, \boldsymbol{y}} & =\left(r^{y_{0}} s^{x_{0}}, \cdots, r^{y_{i}} s^{x_{i}}, \cdots, r^{y_{n-1}} s^{x_{n-1}}\right) \in C .
\end{aligned}
$$


Then

$$
\begin{aligned}
c_{\boldsymbol{a}, \boldsymbol{b}} c_{\boldsymbol{x}, \boldsymbol{y}}= & \left(r^{b_{0}} s^{a_{0}} r^{y_{0}} s^{x_{0}}, \cdots, r^{b_{i}} s^{a_{i}} r^{y_{i}} s^{x_{i}}, \cdots,\right. \\
& \left.r^{b_{n-1}} s^{a_{n-1}} r^{y_{n-1}} s^{x_{n-1}}\right) \\
= & \left(r^{b_{0}+y_{0}\left(1-2 a_{0}\right)} s^{a_{0}+x_{0}}, \cdots, r^{b_{i}+y_{i}\left(1-2 a_{i}\right)} s^{a_{i}+x_{i}}, \cdots,\right. \\
& \left.r^{b_{n-1}+y_{n-1}\left(1-2 a_{n-1}\right)} s^{a_{n-1}+x_{n-1}}\right) .
\end{aligned}
$$

Equation (12) implies

$$
\begin{aligned}
\left(b_{0}+y_{0}\left(1-2 a_{0}\right), \cdots, b_{i}+y_{i}\left(1-2 a_{i}\right)\right. & , \cdots, \\
\left.b_{n-1}+y_{n-1}\left(1-2 a_{n-1}\right)\right) & \in C_{r}
\end{aligned}
$$

or, equivalently,

$$
\begin{aligned}
\left(b_{0}+y_{0}-2 a_{0} y_{0}, \cdots,\right. & b_{i}+y_{i}-2 a_{i} y_{i}, \cdots, \\
& \left.b_{n-1}+y_{n-1}-2 a_{n-1} y_{n-1}\right) \in C_{r}
\end{aligned}
$$

and

$$
\left(a_{0}+x_{0}, \cdots, a_{i}+x_{i}, \cdots, a_{n-1}+x_{n-1}\right) \in C_{s} .
$$

From (13) it follows that

$$
C_{s} \odot 2 C_{r} \in C_{r} .
$$

Conversely, given (10), (11), (13), and (14), it is straightforward to show that (12) holds.

The arguments above hold for both $M$ odd and even. When $M$ is odd 2 is a unit in $Z_{M}$ and hence $2 C_{r}$ is the same as $C_{r}$, since $C_{r}$ is linear.

(Q.E.D.)

In Theorem $1, M$ is an arbitrary integer. When $M$ is a power of 2, say, $M=2^{L-1}$, and $C_{r}$ is decomposable in terms of $L-1$ linear binary codes $C_{1}, C_{2}, \cdots, C_{L-1}$, i.e., can be obtained as

$$
C_{r}=C_{1}+2 C_{2}+\cdots 2^{L-2} C_{L-1}
$$

then Theorem 1 coincides with [1, Theorem 1], the proof of which is straightforward and is contained in [1, proof of Theorem 1]. Whereas [1] is primarily concerned with the case of $M$ being a power of 2 with a restriction that corresponds in our case to the case of $C_{r}$ being a decomposable code, our treatment is for arbitrary $M$, and $C_{r}$ is not necessarily a decomposable code. Our construction can be viewed as a nonbasic multilevel construction [4] with $L$ binary codes among which the last $L-1$ binary codes being related.

Example 1: If $C_{r}=Z_{M}^{n}$, the trivial code consisting of all possible $n$-tuples over $Z_{M}$, and $C_{s}$ any binary linear code or if $C_{r}$ is any linear code over $Z_{M}$ and $C_{s}$ is the repetition code than (11) is satisfied and hence the resulting code is a group code over $D_{M}$.

Example 2: Let

$$
C_{s}=\{000,111\}
$$

and

$$
C_{r}=\{000,220,132,312,200,020,332,112\}
$$

over $Z_{4}$. Then the resulting two-level code is a group code over $D_{4}$ and has the following 16 codewords:

$$
\begin{aligned}
& \left\{\left(r^{0}, r^{0}, r^{0}\right),\left(r^{2}, r^{2}, r^{0}\right),\left(r^{1}, r^{3}, r^{2}\right),\left(r^{3}, r^{1}, r^{2}\right),\right. \\
& \left(r^{2}, r^{0}, r^{0}\right),\left(r^{0}, r^{2}, r^{0}\right),\left(r^{3}, r^{3}, r^{2}\right),\left(r^{1}, r^{1}, r^{2}\right), \\
& (s, s, s),\left(r^{2} s, r^{2} s, s\right),\left(r^{1} s, r^{3} s, r^{2} s\right),\left(r^{3} s, r^{1} s, r^{2} s\right), \\
& \left.\left(r^{2} s, r^{0} s, s\right),\left(s, r^{2} s, s\right),\left(r^{3} s, r^{3} s, r^{2} s\right),\left(r^{1} s, r^{1} s, r^{2} s\right)\right\} .
\end{aligned}
$$

Observe that in this example, $M$ is a power of 2 , but $C_{r}$ is a nondecomposable code.
Example 3: Let

$$
C_{r}=\{0000,1233,3211,0222,1011,2022,3033,2200\}
$$

over $Z_{4}$ and $C_{s}=\{0000,1111\}$. For these codes

$$
C_{s} \odot 2 C_{r}=\{0000,2022\} \subseteq C_{r} .
$$

Therefore, the two-level code $r^{C_{r}} C_{s}$ is a group code over $D_{4}$ and the codewords are

$$
\begin{aligned}
& (111),\left(1, r^{2}, r^{2}, r^{2}\right),\left(r^{3}, r^{2}, r, r\right),\left(r, r^{2}, r^{3}, r^{3}\right), \\
& (r, 1, r, r),\left(r^{2}, r^{2}, 1,1\right),\left(r^{3}, 1, r^{3}, r^{3}\right),\left(r^{2}, 1, r^{2}, r^{2}\right), \\
& (s, s, s, s),\left(s, r^{2} s, r^{2} s, r^{2} s\right),\left(r^{3} s, r^{2} s, r s, r s\right), \\
& \left(r s, r^{2} s, r^{3} s, r^{3} s\right),(r s, s, r s, r s),\left(r^{2} s, r^{2} s, s, s\right), \\
& \left(r^{3} s, s, r^{3} s, r^{3} s\right), \text { and }\left(r^{2} s, s, r^{2} s, r^{2} s\right) .
\end{aligned}
$$

\section{A. Semidirect Product Group Codes}

Since dihedral groups are semidirect product groups, in the first part of the proof of Theorem 1 (11) can be proved using the algebraic condition for two-level group codes over semidirect product groups given by Garello and Benedetto [7, Theorem 2]. Observe that Theorem 1 does not assume linearity of the component codes whereas [7, Theorem 2] does. Given a group $G$ and a normal subgroup $R$ when there exists a group of coset identifiers for the quotient group $S=G / R$, rather than simply a set, $G$ is called the semidirect product of $S$ by $R$. Dihedral groups are semidirect product groups with the normal subgroup generated by the element $r$. An example of a group which is not a semidirect product group is the Quaternion group with eight elements. Stated in the notations of this paper [7, Theorem 2] is "if $s^{C_{s}}$ normalizes $r^{C_{r}}$, i.e.,

$$
c_{\boldsymbol{a}, \boldsymbol{b},-\boldsymbol{a}} \in r^{C_{r}}, \quad \forall \boldsymbol{b} \in C_{r}, \forall \boldsymbol{a} \in C_{s}
$$

then the resulting two-level code is a group code." That the condition given by (11) is the same as (15) can be seen as follows: Since $C_{s}$ is a binary code $\boldsymbol{a}=-\boldsymbol{a}$ in (15), and (15) becomes

$$
c_{\boldsymbol{a}, \boldsymbol{b}, \boldsymbol{a}} \in r^{C_{r}}, \quad \forall \boldsymbol{b} \in C_{r}, \forall \boldsymbol{a} \in C_{s} .
$$

When expressed only in terms of $C_{s}$ and $C_{r}$, (15) becomes $\boldsymbol{b} \odot$ $(1-2 \odot a) \in C_{r}$ or, equivalently,

$$
\boldsymbol{b} \odot 2(-\boldsymbol{a}) \in C_{r}
$$

which in turn is equivalent to (11) for $C_{r}$ being linear $a \in C_{r}$ iff $-\boldsymbol{a} \in C_{r}$.

Moreover, from [7, Theorem 6], it follows that all group codes over dihedral groups are not necessarily obtainable as two-level codes.

\section{Euclidean Distance Properties}

In this section the main results of this correspondence, i.e., the Euclidean distance properties of the signal space code $\mu^{n}(\boldsymbol{C})$ are discussed. It is shown that the ratio of the Hamming distances of the component codes $C_{s}$ and $C_{r}$ provides a good deal of information about the MSED of the signal space code under different matched labelings. In Section IV-A, we restrict the discussion to symmetric labelings and in Section IV-B, the results of symmetric labelings are extended to asymmetric labelings. Results for 6-SPSK and 4-APSK are discussed as special cases.

The following notations are used throughout this section. Fig. 2(a)-(c) illustrates the various distances represented by the notations.

$d_{s} \quad$ minimum Hamming distance of $C_{s}$;

$d_{r} \quad$ minimum Hamming distance of $C_{r}$; 
$d_{\mu} \quad$ MSED of $\mu^{n}(\boldsymbol{C})$ under the matched labeling $\mu$, of $2 M$ SPSK or $2 M$-APSK (e.g., $d_{S L_{\psi^{\prime}}}=$ MSED of $\mu^{n}(\boldsymbol{C})$ when $\mu$ is $\left.S L_{\phi}\right)$;

$\delta_{r} \quad$ Euclidean weight of $\mu(r)$ in $2 M$-SPSK under any labeling $=4 \sin ^{2}(\pi / M)$;

$\delta_{m}$ Euclidean weight of $\mu(s)$ under $m L$ of $2 M$-SPSK $=$ $4 \sin ^{2}[(2 m+1) \pi /(2 M)]$;

$\delta_{\phi} \quad$ Euclidean weight of the signal point nearest to $\mu(e)$ with the asymmetry $\phi$ under any matched labeling $=4 \sin ^{2}[((\pi / M)-|\phi|) / 2]$;

$\delta_{m_{\phi}} \quad$ Euclidean weight of $\mu(s)$ under

$$
m L_{\phi}=4 \sin ^{2}[(((2 m+1) \pi / M)+\phi) / 2] ;
$$

$\delta_{\mu} \quad$ Euclidean weight of the signal point $\mu(s)$ under the matched labeling $\mu$.

Remark 1: Note that $\delta_{\phi}$ and $\delta_{0_{\phi}}$ are not the same. In fact, they are equal for negative values of $\phi$, and $\delta_{0_{\phi}}=\delta_{S L_{\phi}}>\delta_{\phi}$, for positive values of $\phi$. Moreover, $\delta_{\phi}$ with $\phi=0$, i.e., $\delta_{0}$ is same as $\delta_{S L}$.

The following lemma, used often throughout this section, gives a simple lower bound on $d_{m L_{\phi}}$.

Lemma 1:

$$
d_{m L_{\phi}} \geq \min \left\{\delta_{r} d_{r}, \delta_{m_{\phi}} d_{s}, \delta_{\phi} \max \left(d_{r}, d_{s}\right)\right\} .
$$

Proof: From

i) Any signal point in $\mu^{n}(\boldsymbol{C})$ corresponding to a codeword consisting only of $s$-terms (elements of $s^{C_{s}}$ ) has Euclidean weight at least $\delta_{m \phi} d_{s}$, and a signal point exists with weight equal to $\delta_{m \hat{\phi}} d_{s}$.

ii) Any signal point in $\mu^{n}(\boldsymbol{C})$ corresponding to a codeword consisting only of $r$-power terms (elements of $r^{C_{r}}$ ) has Euclidean weight at least $\delta_{r} d_{r}$.

iii) Any signal point in $\mu^{n}(\boldsymbol{C})$ corresponding to a codeword consisting of at least one term of the form $r^{i} s, i \neq 0$, has Euclidean weight at least $\delta_{\phi} \max \left(d_{r}, d_{s}\right)$

the inequality (16) follows.

Q.E.D.

Remark 2: If

$$
\min \left\{\delta_{r} d_{r}, \delta_{m_{\phi}} d_{s}, \delta_{\phi} \max \left(d_{r}, d_{s}\right)\right\}=\delta_{m_{\phi^{\prime}}} d_{s}
$$

then $d_{m L_{\phi}}=\delta_{m_{\phi}} d_{s}$, in view of i) above, i.e., equality holds in (16). Specializing (16) for $m L$, to be used for symmetric labelings

$$
d_{m L} \geq \min \left\{\delta_{r} d_{r}, \delta_{m} d_{s}, \delta_{0} \max \left(d_{r}, d_{s}\right)\right\}
$$

Since comparison of different labelings will be made with the corresponding standard labelings, we specialize (16) and (17), respectively, for later use, to $S L_{\phi}$ and $S L$, and a few further special cases corresponding to $d_{s}<d_{r}$ or $d_{s}>d_{r}$, as follows:

Specializing (16) to $S L_{\phi}$

$$
d_{S L_{\phi}} \geq \min \left\{\delta_{r} d_{r}, \delta_{0_{\phi}} d_{s}, \delta_{\phi} \max \left(d_{r}, d_{s}\right)\right\} .
$$

If $d_{s}<d_{r}$ then

$$
d_{S L_{\phi}} \geq\left\{\delta_{\phi} d_{r}, \delta_{0_{\phi}} d_{s}\right\}
$$

and, in addition, if $\phi<0$, then, using Remark 1

$$
d_{S L_{\phi}}=\delta_{\phi} d_{s} .
$$

If $d_{s}>d_{r}$ then

$$
d_{S L_{\phi}} \geq\left\{\delta_{r} d_{r}, \delta_{\phi} d_{s}\right\}
$$

and in addition if $d_{s} / d_{r}<\delta_{r} / \delta_{\phi}$, then

$$
d_{S L_{\phi}}=\delta_{\phi} d_{s} \text {. }
$$

Specializing (17) to $S L$

$$
d_{S L} \geq \min \left\{\delta_{r} d_{r}, \delta_{0} d_{s}, \delta_{0} \max \left(d_{r}, d_{s}\right)\right\}
$$

If $d_{s}<d_{r}$ then

$$
d_{S L}=\delta_{0} d_{s} .
$$

If $d_{s}>d_{r}$ and $d_{s} / d_{r} \leq \delta_{r} / \delta_{0}$, then

$$
d_{S L} \geq \delta_{0} d_{s}
$$

which is well known.

\section{A. Euclidean Distance Properties for SPSK Modulation}

This subsection deals with Euclidean distance properties of $\mu^{n}(\boldsymbol{C})$ under symmetric labelings. Throughout this subsection a labeling will always mean symmetric labeling. Fig. 3(a) shows an axis used to identify a point corresponding to all possible values of $d_{s} / d_{r}$. Given a pair of component codes their ratio of Hamming distances uniquely defines a point on this axis. The points corresponding to $4 / \delta_{0}, \delta_{r} / \delta_{0}$, and different values of $\delta_{0} / \delta_{m}$ marked on the axis demarcate various regions to be discussed. Note that the point $\delta_{0} / \delta_{m}$ shifts to the left or right according to increase or decrease in the Lee weight of $m$.

The entire $d_{s} / d_{r}$-axis in Fig. 3(a) is divided into two corresponding to the two major cases discussed: 1) $d_{s}<d_{r}$, i.e., the axis to the left of the point $d_{s} / d_{r}=1$ (Theorem 2), and 2) $d_{s}>d_{r}$, i.e., the axis to the right of the point $d_{s} / d_{r}=1$ (Theorem 3). Each case is further divided into three subcases identified as Regions I, II, and III for the first case and Regions IV, V, and VI for the second case. Corollaries 2.1 and 2.2, respectively, deal exclusively with Region I and Region III.

Theorem 2 (Case $d_{s}<d_{r}$-Region I, II and III in Fig. 3(a)):

i) If, for some $m$

$$
0<d_{s} / d \hat{r} \leq \delta_{0} / \delta_{m}
$$

then $m L$ gives larger MSED compared to all $m L$, where Lee $(m)<$ Lee $(\boldsymbol{m})$.

ii) If, for some $\boldsymbol{m}$

$$
\delta_{0} / \delta_{m} \leq d_{s} / d_{r}<1
$$

then all $m L$ such that Lee $(m) \geq$ Lee $(\boldsymbol{m})$, gives larger MSED than $S L$, where Lee $(m)$ denotes Lee weight of $m$.

iii) If $d_{s} / d_{r}<1$, then there exist $m L$ which gives larger MSED than $S L$.

Proof:

i) Using (18) in (17) and in view of Remark 2, we have $d_{\boldsymbol{m} L}=$ $\delta_{\boldsymbol{m}} d_{s}$. Since $\delta_{m}<\delta_{\boldsymbol{m}}$ if Lee $(m)<\operatorname{Lee}(\boldsymbol{m})$, it follows that $d_{m L}<d_{\boldsymbol{m} L}$ for all $m$, such that Lee $(m)<$ Lee $(\boldsymbol{m})$.

ii) From (17b) we have $d_{S L}=\delta_{0} d_{s}$. Using (19), (17) becomes

$$
d_{\boldsymbol{m} L} \geq \min \left\{\delta_{0} d_{r}, \delta_{\boldsymbol{m}} d_{s}\right\}=\delta_{0} d_{r} .
$$

Since $d_{r}>d_{s}$, we have $d_{\boldsymbol{m} L}>d_{S L}$ for all $\boldsymbol{m}$ such that (19) is satisfied. The values of $m$ that satisfy (19) are those for which Lee $(m) \geq$ Lee $(\boldsymbol{m})$.

iii) Follows from combining parts i) and ii).

Q.E.D.

Theorem 2 part i) states that given the value $d_{s} / d_{r}$, the labeling corresponding to the closest point of the value to the right of it, say 


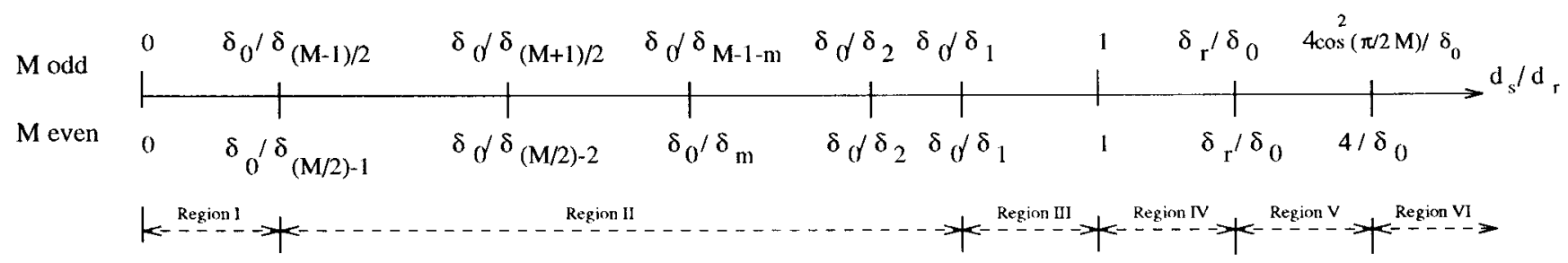

(a)

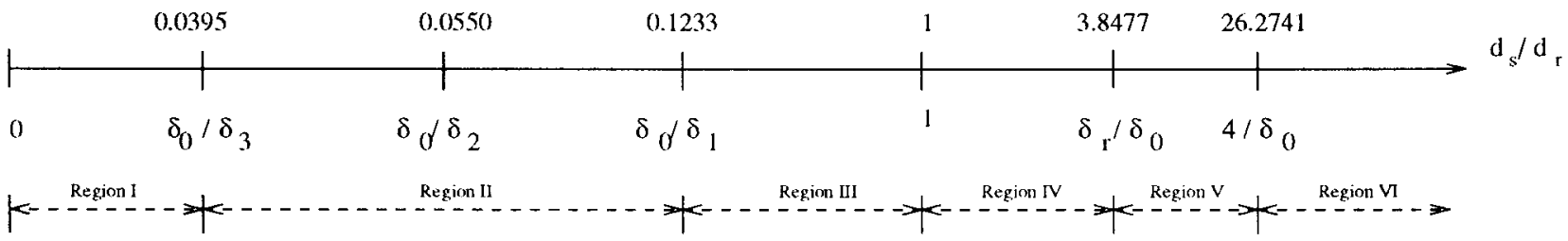

(b)

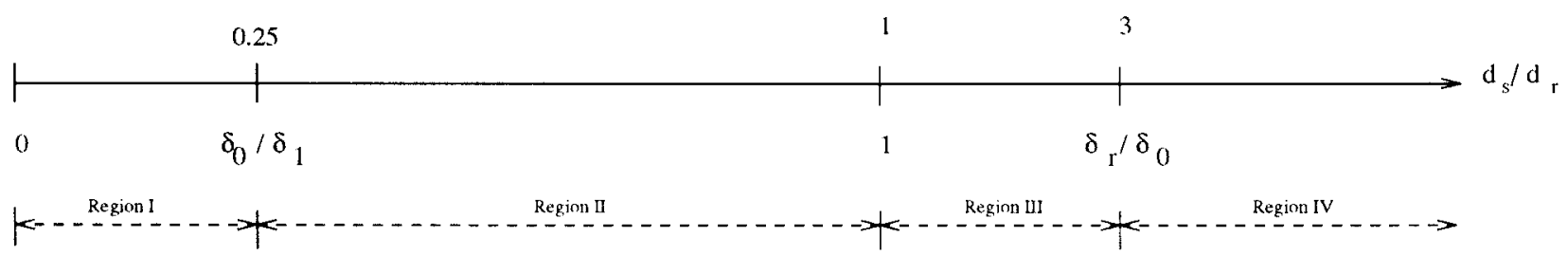

(c)

Fig. 3. (a) $d_{s} / d_{r}$-axis for $2 M$-SPSK modulation. (b) $d_{s} / d_{r}$-axis for 16-PSK modulation. (c) $d_{s} / d_{r}$-axis for 6-PSK modulation.

$\boldsymbol{m} L$, performs better than all other labelings on the right and Theorem 2 part ii) states that all labelings that are to the left of $\boldsymbol{m} L$, perform better than $S L$.

The following two corollaries follow, respectively, from Theorem 2 parts i) and ii).

Corollary 2.1 (Region I in Fig. 3(a)): If $d_{s} / d_{r}$ satisfies

$$
0<d_{s} / d_{r} \leq \delta_{0} / \delta_{M D L}
$$

then $M D L$ gives larger MSED than any other matched labeling.

Corollary 2.2 (Region III in Fig. 3(a)): If $d_{s} / d_{r}$ satisfies $\delta_{0} / \delta_{1}<$ $d_{s} / d_{r}<1$, than any matched labeling other than $S L$ will give larger MSED than $S L$.

Several examples are given for 16-PSK signal set, i.e., $M=8$. Accordingly, Fig. 3(a) is redrawn as Fig. 3(b), for the specific value of $M=8$ with specific values demarcating the regions marked. Fig. 2(c) shows the 16-SPSK signal set with relevant parameters marked.

Example 4: Let $C_{s}$ be the length 20 binary cyclic code generated by $1+x^{2}$ and $C_{r}$ be the length 20 code over $Z_{8}$ with generator matrix $\left[44 \ldots 4\right.$ 4]. Since $d_{s} / d_{r}=2 / 20=0.1<\delta_{0} / \delta_{1}=0.123309$, this case corresponds to Region II in Fig. 3(b). We have

$$
\begin{aligned}
& d_{3 L}=3.044818 \quad d_{2 L}=5.530732 \quad d_{1 L}=2.46926 \\
& d_{0 L}=0.3044818
\end{aligned}
$$

from which it is seen that $m=2$ corresponds to the best labeling.

Example 5: Let $C_{s}$ be the length 30 binary code consisting of the following four codewords:

$$
(000 \ldots 00)(100 \ldots 00) \quad(011 \ldots 11) \quad(111 \ldots 11)
$$

and $C_{r}$ be the length 30 code over $Z_{8}$ whose generator matrix is $\left[\begin{array}{llllllllllllllllllllllll}0 & 1 & 1 & . & . & . & 1 & 1 & 1 & 1 & 1 & . & . & . & 1 & 1 & 0 & 0 & 0 & . & . & 0 & 0 \\ 0 & 0 & 0 & . & . & . & 0 & 0 & 1 & 1 & 1 & . & . & . & 1 & 1 & 1 & 1 & 1 & . & . & . & 1 & 1\end{array}\right]$.
Since $d_{s} / d_{r}=1 / 19=0.0526315<\delta_{0} / \delta_{2}=0.05505$, this case corresponds to Region II in Fig. 3(a). Moreover, $19 \delta_{0}=2.8925771$, and $d_{3 L}=2.8925771, d_{2 L}=2.765366, d_{1 L}=1.23463, d_{0 L}=$ 0.1522409. It is clear that $m=2$ gives larger MSED than $m=1$ and $m=0(S L)$ and lesser than $m=3,(M D L)$.

Example 6: This example illustrates Corollary 2.1. Let $C_{s}=Z_{2}^{30}$ and $C_{r}$ be the length 30 code over $Z_{8}$ with generator matrix

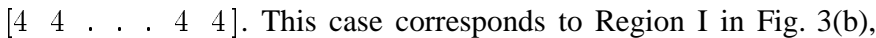
since $d_{s} / d_{r}=1 / 30=0.0333333<\delta_{0} / \delta_{M D L}=0.039566137$. In this case

$d_{3 L}=3.84776 \quad d_{2 L}=2.765366 \quad d_{1 L}=1.23463$

$$
\text { and } d_{0 L}=0.1522409 \text {. }
$$

Moreover, $\left(r^{4} s, r^{4} s, \cdots, r^{4} s\right)$ has Euclidean weight $=30 \delta_{0}=$ 4.567228. Obviously, $M D L$ gives the best performance. If asymmetry is introduced then $\delta_{3}$ will increase and the figure $30 \delta_{0}=4.567228$ will decrease. The best asymmetry is that which equals these two. In the following subsection we pursue this example further by introducing the angle of asymmetry and improve the MSED.

Example 7: This illustrates Corollary 2.2. Let $C_{s}$ be the length 33 binary code consisting of the following four codewords:

$$
\left(\begin{array}{lllllllll}
0 & 0 & 0 & 0 & . & . & 0 & 0
\end{array}\right) \quad\left(\begin{array}{lllllllll}
1 & 1 & 0 & 0 & . & . & 0 & 0
\end{array}\right)
$$

and $C_{r}$ be the length 33 code over $Z_{8}$ with generator matrix shown at the bottom of the next page. Since $d_{s} / d_{r}=2 / 20=0.1<\delta_{0} / \delta_{1}=$ 0.123309 , this case corresponds to Region III in Fig. 3(b). We have $20 \delta_{0}=3.044818$, and

$$
d_{3 L}=3.044818 \quad d_{2 L}=3.044818 \quad d_{1 L}=2.46926
$$

$$
d_{0 L}=0.3044818 \text {. }
$$

It is clear that all the three labelings $1 L, 2 L$, and $3 L$ are better than $S L$. 


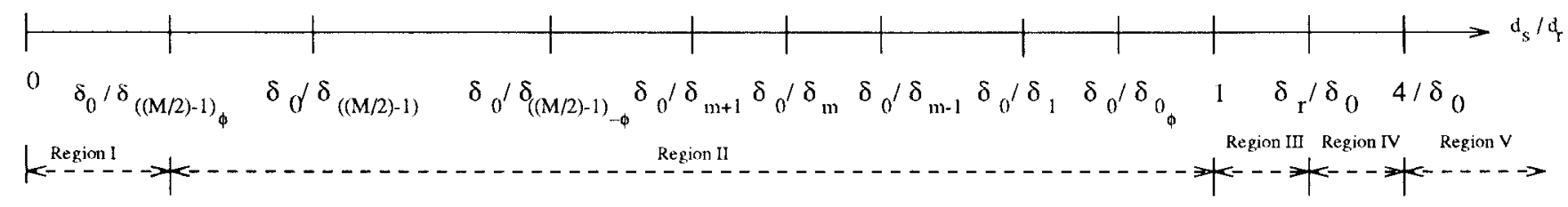

(a)

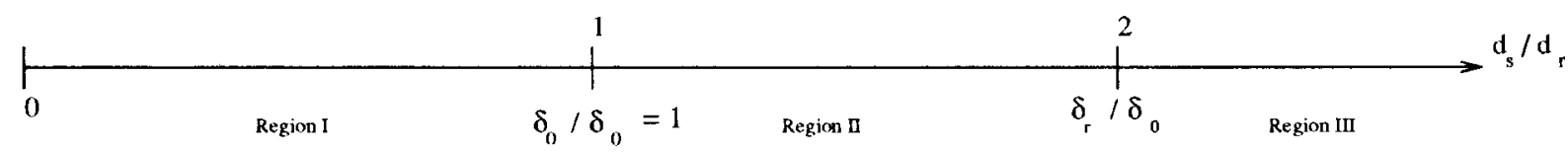

(b)

Fig. 4. (a) $d_{s} / d_{r}$-axis for $2 M$-APSK modulation with angle of asymmetry $\phi, 0<\phi<\pi / 2 M$ (shown only for $M$ even; for $M$ odd it is similar). (b) $d_{s} / d_{r}$-axis for 4-PSK modulation.

Theorem 3 (Case $d_{s}>d_{r}-$ Region IV, V, and VI in Fig. 3(a)):

i) If $d_{s} / d_{r}$ is in Region IV in Fig. 3(a), i.e., if $1<d_{s} / d_{r}<$ $\delta_{r} / \delta_{0}$, then for all $m$

$$
d_{m L} \geq d_{S L}
$$

and strict inequality holds for those values of $m$ for which if $C_{r}$ contains a codeword, say $c$, of Hamming weight $d_{s}$ with nonzero components in those locations in which a minimum weight vector of $C_{s}$ has nonzero components then at least one nonzero component of $\boldsymbol{c}$ does not belong to $\{M-1-m, M-m\}$. Moreover, if a minimum weight vector of $C_{s}$ is present also in $C_{r}$ then all labelings will give the same MSED.

ii) If $d_{s} / d_{r}$ is in Region V, in Fig. 3(a), i.e., if $\delta_{r} / \delta_{0}<d_{s} / d_{r}<$ $\lambda / \delta_{0}$, then $d_{m L} \geq d_{S L}$, where $\lambda=4$ if $M$ is even and

$$
\lambda=4 \cos ^{2}(\pi / 2 M)=\text { Euclidean weight of } \mu\left(r^{\frac{M-1}{2}}\right)
$$

if $M$ is odd.

iii) If $d_{s} / d_{r}$ is in Region VI in Fig. 3(a), i.e., if $d_{s} / d_{r}>\lambda / \delta_{0}$, then MSED is same for all matched labelings.

The proof of Theorem 3 is given in Appendix I. It follows from Theorem 3 that if the ratio of the Hamming distances of the component code lies in Region VI then all labelings lead to the same MSED, i.e., there is no preferred labeling; if the ratio lies in Region $\mathrm{V}$ then we are able to say that all nonstandard labelings perform as good as or better than standard labeling, but we are unable to identify situations with strictly better performance; if the ratio lies in Region IV we not only show that all nonstandard labelings perform at least as good as standard labelings but identify situations where they perform strictly better and situations where they perform exactly as good as standard labelings. We illustrate these in the following two examples.

Example 9: Let $C_{s}=\{0000,1111\}$ and

$$
C_{r}=\{0000,1233,2466,3611,4044,5277,6422,7655\}
$$

a linear code over $Z_{8}$ with generator matrix [ [ $\left.\begin{array}{llll}1 & 2 & 3 & 3\end{array}\right]$. This corresponds to Region IV in Fig. 3(b), since $1<d_{s} / d_{r}<\delta_{r} / \delta_{0}=3.847715$. Moreover,

$$
\begin{aligned}
& d_{S L}=0.608967 \text { and } d_{1 L}=4.93852 \\
& d_{2 L}=5.3868729 \text { and } d_{M D L}=4.304984 .
\end{aligned}
$$

All labelings have larger MSED than $S L$, as expected since $C_{r}$ does not contain a codeword of the form that will give equality as discussed in the proof of Theorem 3 .

If $C_{r}$ is changed to

$$
\left[\begin{array}{llll}
1 & 2 & 3 & 3 \\
2 & 2 & 0 & 0
\end{array}\right]
$$

then $d_{s} / d_{r}=2$, and hence still corresponds to Region IV. The MSED under various labelings are

$d_{S L}=0.608967 \quad d_{1 L}=1.6906 \quad d_{2 L}=1.6906$ and $d_{M D L}=0.608967$.

In this case $C_{r}$ contains the codeword (5455) which leads to $d_{3 L}=d_{M D L}=d_{S L}$. Instead, if $C_{r}$ is changed to

$$
\left[\begin{array}{llll}
1 & 2 & 3 & 3 \\
1 & 1 & 0 & 0
\end{array}\right]
$$

then $d_{s} / d_{r}$ remains at 2 and the MSED under different labelings are $d_{S L}=0.608967 \quad d_{1 L}=1.1716 \quad d_{2 L}=0.608967$ and $d_{M D L}=0.608967$.

In this case, $(6555) \in C_{r}$ leads to the equality $d_{2 L}=d_{S L}$ and $(5455) \in C_{r}$ maintains the equality $d_{3 L}=d_{M D L}=d_{S L}$. Further, if $C_{r}$ is changed to

$$
\left[\begin{array}{llll}
1 & 2 & 3 & 3 \\
1 & 1 & 1 & 1
\end{array}\right]
$$

then $d_{s} / d_{r}$ becomes 4 and the MSED under all labelings is $d_{S L}=$ 0.608967 , including $M D L$ in accordance with Corollary 1 above.

Example 10: Let $C_{s}$ be the length 8 repetition code and $C_{r}$ be the cyclic code over $Z_{8}$ with generator polynomial $\left(1+x^{2}\right)$. In this case

$$
\delta_{r} / \delta_{0}=3.847715<d_{s} / d_{r}=4<4 / \delta_{0}=26.27413115
$$

and hence this example corresponds to Region V in Fig. 3(b). The MSED is 1.1716 corresponding to the codeword $(r, e, r, e, e, \cdots, e)$ which is $2 \delta_{r}=2(0.5858)$. Note that $8 \delta_{0}=1.2179$ hence the MSED is achieved by an $r$-power-only codeword and hence the same for all labelings.

When considering the special case of 6-SPSK, the above results take a simpler form, as given in the following theorem. Notice that

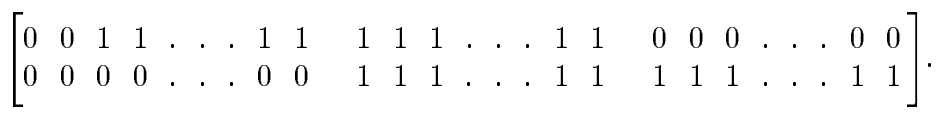


for 6-SPSK there are only two labelings, $M D L$ and $S L$. This means the situations corresponding to Regions II and V of the general setting of Fig. 3(a) do not arise since there is only one labeling to the left of $d_{s} / d_{r}=1$ which is MDL.

Theorem 4 (6-SPSK case-Fig. 3(c)):

i) If $d_{s} / d_{r}<1$ (Regions I and II in Fig. 3(c)) then $d_{M D L}>d_{S L}$.

ii) If $1<d_{s} / d_{r}<3$ (Region III in Fig. 3(c)) then $d_{M D L} \geq d_{S L}$ with strict inequality if $C_{r}$ does not contain a codeword of weight $d_{s}$ with nonzero components exactly in the same locations as those of a minimum-weight codeword of $C_{s}$.

iii) If $d_{s} / d_{r}>3$ (Region IV in Fig. 3(c)) then the MSED is the same for all matched labelings.

Proof: i), ii), and iii) follow from specializing to $M=3$, respectively, Theorem 2 part iii), Theorem 3 part i), and Theorem 3 part iii).

\section{B. Euclidean Distance Properties for APSK Modulation}

In this subsection we discuss the Euclidean distance properties of $\mu^{n}(C)$ under asymmetric labelings. Like Fig. 3(a) for SPSK in the previous subsection, the $d_{s} / d_{r}$-axis shown in Fig. 4(a) will be used to identify the different regions discussed in the theorems of this subsection. Note that the point $\delta_{0} / \delta_{m}$ shifts by at most $\pi / 2 M$ to the left or right according to a positive or a negative angle of asymmetry if $0<\mu(s)<\pi$, and in the opposite way if $\pi<\mu(s)<2 \pi$.

As in the case of SPSK in the previous section, we also consider two major cases for APSK: 1) $d_{s}<d_{r}$ (Theorem 5 and the special case 4-APSK in Theorem 6) and 2) $d_{s}>d_{r}$ (Theorem 7). The first case is further divided into two subcases identified as Regions I and II (Fig. 4(a)) and the second into three subcases identified as Regions III, IV, and V.

Theorem 5 (Case: $d_{s}<d_{r}-$ Regions I and II in Fig. 4(a)):

1) For all values of $\boldsymbol{m}$, satisfying $d_{s} / d_{r}<\delta_{0} / \delta_{\boldsymbol{m}}$, the MSED of $\mu^{n}(\boldsymbol{C})$ monotonically increases (from the MSED corresponding to $\boldsymbol{m} L$ ) with respect to the angle of asymmetry $0<\phi \leq \Phi$, where $\Phi$ is given by (21) at the bottom of this page, unless $M$ is odd and $\boldsymbol{m}=(M-1) / 2$, in which case $\Phi=0$. For $\phi<0$, the MSED of $\mu^{n}(\boldsymbol{C})$ decreases.

2) If $m L$ satisfies $\delta_{0} / \delta_{m}<d_{s} / d_{r}<1$, then $d_{m L_{\phi}}>d_{S L_{\phi}}$ for all values of $\phi$, in the range

$$
\begin{aligned}
& -2 \tan ^{-1}\left[\frac{\sqrt{d_{s} / d_{r}} \sin ((2 m+1) \pi / 2 M)-\sin (\pi / 2 M)}{\sqrt{d_{s} / d_{r}} \cos ((2 m+1) \pi / 2 M)-\cos (\pi / 2 M)}\right] \\
& <\phi<(\pi / M) \text {. }
\end{aligned}
$$

\section{Proof: Given in Appendix II.}

Remark 3: Note that the value of $\Phi$, given by (21), depends on $\boldsymbol{m}$.

The essence of part i) of Theorem 5 is the following: In comparison with every SPSK labeling, say $m L$, when $d_{s} / d_{r}<\delta_{0} / \delta_{m}$, then the MSED increases due to asymmetry up to some fixed positive angle (the image of $s$ is moved away from $(1,0)$ ) given by (21) and this fixed angle is a function of $\boldsymbol{m}$. Notice that nothing is said regarding the increase or decrease for positive angles more than this fixed angle. For negative angles of asymmetry, the MSED decreases. The essence of part ii) of Theorem 5 is that the superiority of $\boldsymbol{m} L$ over $S L$ for
SPSK case proved in part ii) of Theorem 2 continues to hold in the case of APSK also for angles in the range given by (22).

Example 5 (continued): If $2 L$ is chosen, then the performance can be improved by introducing the asymmetry. As per Theorem 5 , the improvement is guaranteed up to $\Phi=0.4485548^{\circ}$, in which case we get $d_{2 L_{\phi}}=\delta_{2 L_{\phi}}=2.7798089$ and $19 \delta_{\phi}=2.7798092$.

Observe that in the example above, further increase will decrease the MSED of the code since $d_{2 L_{\phi}}$ will then be $19 \delta_{\Phi}$. Whereas Theorem 5 does not say anything regarding the decrease or further increase of the MSED as $\Phi$ is increased, the following corollary gives conditions for decrease.

Corollary 5.1: i) In Theorem 5 part i) if a minimum-weight vector $\boldsymbol{b} \in C_{r}$ consists of the nonzero value $M / 2$ only and there is $\boldsymbol{a} \in C_{s}$, with nonzeros in exactly same locations as that of $\boldsymbol{b}$, then $d_{\boldsymbol{m} L_{\Phi}}$ will decrease for further increase in $\phi$.

ii) In Theorem 5 part i), if $m$ is even, $\boldsymbol{m}=M / 2-1$, and if a minimum-weight vector $\boldsymbol{b} \in C_{r}$ consists of the nonzero value $M / 2$ only and there is $\boldsymbol{a} \in C_{s}$, with nonzeros in exactly the same locations as that of $\boldsymbol{b}$, then $\boldsymbol{m} L_{\Phi}$ gives the largest MSED compared to any matched labeling, i.e., $\boldsymbol{m} L_{\Phi}$ is the best matched labeling.

Proof: i) The signal point $\mu^{n}(\boldsymbol{a}, \boldsymbol{b})$ has Euclidean weight $d_{r} \delta_{\phi}$. Therefore, for $\phi>\Phi$ given by (21) the MSED is $d_{r} \delta_{0_{\omega}}$, which decreases from $d_{\boldsymbol{m} L_{\Phi}}$ as $\phi$ increases.

ii) The signal point $\mu^{n}(\boldsymbol{a}, \boldsymbol{b})$ has Euclidean weight $d_{r} \delta_{0_{\phi}}$. Therefore, for $\phi>\Phi$ given by (21) the MSED is $d_{r} \delta_{\phi}$, which decreases from $d_{M D L_{\Phi}}$ as $\phi$ increases.

Q.E.D.

Example 6 (continued): Now using (21), the angle of asymmetry if $\phi=1.8064609^{\circ}, \delta_{M D L_{\phi}}=3.8709678$, and $30 \delta_{0_{\alpha}}=3.870967$. Note that $\delta_{M D L_{\alpha}}$ increases and $30 \delta_{0_{\phi}}$ decreases as $\phi$ increases and the point that makes these two equal is the best angle. Observe that this example satisfies the condition in part ii) of Corollary 5.1 above and hence $\phi=1.8064609^{\circ}$ corresponds to the best labeling.

Corollary 5.1 leads to a simple characterization and identification of the best labeling for 4-APSK case if $d_{s}<d_{r}$.

Theorem 6 (4-APSK Case-Fig. 4(b)):

i) For the case $M=2$, the condition $C_{s} \odot 2 C_{r} \subseteq C_{r}$ follows from the linearity of the component codes and hence is redundant.

ii) If $d_{s} / d_{r}<1$ (Region I in Fig. 4(b)) monotonic increase in MSED from that corresponding to SPSK is guaranteed up to an angle $\Phi$, where

$$
\Phi=2 \tan ^{-1}\left[\left(1-\sqrt{d_{s} / d_{r}}\right) /\left(1+\sqrt{d_{s} / d_{r}}\right)\right] .
$$

iii) If a minimum-weight vector of $C_{r}$ is present in $C_{s}$ then $\boldsymbol{m} L_{\Phi}$ gives the largest MSED compared to any matched labeling.

Proof: i) Follows from the fact that when $M=2,2 C_{r}=\mathbf{0}$, which is always in $C_{s}$ since it is linear.

ii) The only labeling for 4-PSK is $M D L$. Substituting $M=2$ in (21) gives (23).

iii) Follows from substituting $M=2$ in part ii) of Corollary 5.1.

Q.E.D.

Example 11:

Case i) Let $C_{s}=\{000,101,110,011\}$ and $C_{r}=\{000,111\}$. For this pair of component codes $d_{s} / d_{r}=2 / 3$ and the angle

$$
\Phi=2 \tan ^{-1}\left[\frac{\sin (\pi / 2 M)-\sqrt{d_{s} / d_{r}} \sin ((\boldsymbol{m} \pi / M)+(\pi / 2 M))}{\sqrt{d_{s} / d_{r}} \cos ((\boldsymbol{m} \pi / M)+(\pi / 2 M))+\cos (\pi / 2 M)}\right]
$$


given by (23) is $\Phi=11.536965^{\circ}$, and the corresponding MSED is $d_{M D L_{\Phi}}=4.8$. The minimum-weight vector of $C_{r}$ is not present in $C_{s}$ and hence this MSED is not the best. For instance, $\Phi=20^{\circ}$ gives MSED 5.3680806.

Case ii) Let $C_{s}=Z_{2}^{3}$ and $C_{r}=\{000,011,101,110\}$. For this pair of component codes $d_{s} / d_{r}=1 / 2$ and the angle given by (23) is $\Phi=19.471227^{\circ}$, and the corresponding MSED is $d_{M D L_{\Phi}}=2.666666$. All the minimum-weight vectors of $C_{r}$ are present in $C_{s}$ and hence this MSED is the best. It is easily verified by inspection that for $\delta_{\Phi}=1.333333$ the codeword $(r s, r s, e)$ has Euclidean weight $2 \delta_{\Phi}=2.666666$ which will decrease for any increase in the value of $\Phi$, thus decreasing the MSED of the code.

Case iii) Let $C_{s}=Z_{2}^{3}$ and $C_{r}=\{000,111\}$. In this case, $d_{s} / d_{r}=1 / 3$ and the corresponding value is $\Phi=29.999998^{\circ}$, giving the MSED $d_{M D L_{\Phi}}=3$. The minimum weight vector $(111)$ of $C_{r}$ is present in $C_{s}$ and hence this MSED is the best. It is verified by seeing that $\delta_{\Phi}=1$ and the codeword $(r s, r s, r s)$ has Euclidean weight $3 \delta_{\Phi}=3$ which will decrease for any increase in the value of $\Phi$, thus decreasing the MSED of the code.

Theorem 7 (Case $d_{s}>d_{r}$ ):

i) If $d_{s} / d_{r}$ is in Region III in Fig. 4(a), i.e., if $1 \leq d_{s} / d_{r}<\delta_{r} / \delta_{0}$, then for $-\pi / M<\phi<\pi / M$, we have

$$
d_{m L_{\phi}} \geq d_{S L_{\phi}}
$$

with strict inequality holding for those values of $m$ for which if $C_{r}$ contains a codeword, say $\boldsymbol{c}$, of Hamming weight $d_{s}$ with nonzero components in those locations in which a minimumweight vector of $C_{s}$ has nonzero components then at least one nonzero component of $\boldsymbol{c}$ is not $M-1-m$, if $\phi>0$, and is not $M-m$, if $\phi<0$.

ii) If $d_{s} / d_{r}$ is in Region IV in Fig. 4(a), i.e., if $\delta_{r} / \delta_{0}<d_{s} / d_{r}<$ $4 / \delta_{0}$, then for all values of $\phi$ in the range

$$
|\phi|<\pi / M-2 \sin ^{-1}\left[\sqrt{d_{s} / d_{r}} \sin (\pi / M)\right]
$$

we have

$$
d_{m L_{\phi}} \geq d_{S L_{\phi}}
$$

iii) If $d_{s} / d_{r}$ is in Region $\mathrm{V}$ in Fig. 4(a), i.e., if $d_{s} / d_{r}>4 / \delta_{0}$, then for all values of $\phi$, where

$$
|\phi|<\pi / M-2 \sin ^{-1}\left(\sqrt{d_{r} / d_{s}}\right)
$$

$d_{m L_{\omega}}$ is independent of $m$, i.e., MSED is the same for all matched labelings satisfying (25).

Proof: Given in Appendix III.

Corollary 7.1: In Theorem 7 part i), for some $m$ if $(m, M)=1$ and equality is achieved in (34) in Appendix III, then the all-1 vector belongs to $C_{r}$, and conversely. Hence, if the all-1 vector does not belong to $C_{r}$, then for all $m$ relatively prime to $M$, strict inequality holds in (34).

Example 9 (continuation): By introducing $\phi=2.5^{\circ}$ angle of symmetry the calculated MSED for various labelings are

$$
\begin{aligned}
& d_{S L_{\phi}}=0.4824588 \quad d_{1 L_{\phi}}=0.6159982 \quad d_{2 L_{\phi}}=1.1716 \\
& \text { and } d_{3 L_{\phi}}=0.5492285 \text {. }
\end{aligned}
$$

It is seen that all matched labelings give larger MSED compared to $S L_{\phi}$ as per Theorem 7 part i).

\section{Phase Rotational Invariance}

For $L$-level multilevel codes with binary component codes phase invariance properties have been reported in [3]. In this section we consider the phase rotational invariance property of the two-level group codes over $D_{M}$.

For $m L_{\phi}$ of a $2 M$-APSK signal set, a codeword

$$
\left(r^{y_{0}} s^{x_{0}}, r^{y_{1}} s^{x_{1}}, \cdots, r^{y_{i}} s^{x_{i}}, \cdots, r^{y_{n-1}} s^{x_{n-1}}\right) \in C
$$

is mapped onto the point

$$
\begin{gathered}
\left(\exp \sqrt{-1}\left[x_{0}\left((2 m+1) \frac{\pi}{M}+\phi\right)+y_{0} \frac{2 \pi}{M}\right], \cdots,\right. \\
\exp \sqrt{-1}\left[x_{i}\left((2 m+1) \frac{\pi}{M}+\phi\right)+y_{i} \frac{2 \pi}{M}\right], \cdots, \\
\left.\exp \sqrt{-1}\left[x_{n-1}\left((2 m+1) \frac{\pi}{M}+\phi\right)+y_{n-1} \frac{2 \pi}{M}\right]\right)
\end{gathered}
$$

in $2 n$-dimensional space. There is a one-to-one correspondence between codewords and $2 n$-dimensional points given by (26) and (27). The code is said to be rotationally invariant to angle $\theta$, if whenever (27) is a signal point for a codeword in $C$, then the vector corresponding to the expression at the bottom of this page is a codeword in $C$. Theorem 8 gives the conditions on the component codes for a two-level group code over $D_{M}$ to be invariant to different angles of rotations including the minimum angle.

\section{Theorem 8:}

a) For $m L$ of a $2 M$-SPSK signal set and $m L_{\phi}$ of a $2 M$-APSK signal set, $\boldsymbol{C}=r^{C_{r}} s^{C_{s}}$ is invariant to

i) $k(2 \pi / M)$, rotations where $k$ divides $M$, iff the all- $k$ vector $(k, k, \cdots, k) \in C_{r}$

ii) $k(2 \pi / M)$, rotations where $k$ and $M$ are relatively prime, iff the all-1 vector $(1,1, \cdots, 1) \in C_{r}$.

b) For $m L$ of a $2 M$-SPSK signal set $C$ is invariant to $\pi / M$ rotations iff the all-1 vector is present in $C_{r}$ and $C_{s}$.

Proof: a) i) Let $M / K=\lambda$. Then replacing $y_{i}$ by $y_{i}+\lambda$, $i=0,1, \cdots, n-1$, in (26) corresponds to $\theta$ in (27) getting replaced by $\lambda(2 \pi / M)$, and conversely.

ii) If $k$ and $M$ are relatively prime, then $k(2 \pi / M)$ rotations can be obtained by $k$ successive $2 \pi / M$ rotations and $2 \pi / M$ rotations can be obtained by $u$ successive $k(2 \pi / M)$ rotations where $u$ is given by $u k+\nu M=1$ (Bezout's Theorem). Hence it is sufficient to consider $2 \pi / M$ rotations only for which the all-1 vector $(1,1, \cdots, 1)$ should be in $C_{r}$ which follows from i) with $\lambda=M$.

b) Suppose the all-1 vector is present in both $C_{r}$ and $C_{s}$. The presence of the all-1 vector in $C_{s}$ guarantees rotational invariance by $(2 m+1) \pi / M=m(2 \pi / M)+\pi / M$. The presence of the all1 vector in $C_{r}$ guarantees rotational invariance by all multiples of $2 \pi / M$, including $-m(2 \pi / M)$. Clearly, rotational invariance for both $m(2 \pi / M)+\pi / M$ and $-m(2 \pi / M)$ implies rotational invariance for $\pi / M$. The converse is straightforward.

Q.E.D.

Note that the minimal angle of rotational invariance for $2 M$-SPSK is $\pi / M$ whereas for $2 M$-APSK is $2 \pi / M$.

$$
\begin{array}{r}
\left(\exp \sqrt{-1}\left[x_{0}\left((2 m+1) \frac{\pi}{M}+\phi\right)+y_{0} \frac{2 \pi}{M}+\theta\right], \cdots, \exp \sqrt{-1}\left[x_{1}\left((2 m+1) \frac{\pi}{M}+\phi\right)+y_{i} \frac{2 \pi}{M}+\theta\right], \cdots\right. \\
\left.\quad \exp \sqrt{-1}\left[x_{n-1}\left((2 m+1) \frac{\pi}{M}+\phi\right)+y_{n-1} \frac{2 \pi}{M}+\theta\right]\right)
\end{array}
$$




\section{DISCUSSION}

Group codes over dihedral groups with $2 M$ elements which are constructible as two-level codes with component codes from a binary code and a linear code over appropriate residue class integer ring modulo $M$ have been studied. Block-coded modulation scheme using both $2 M$-SPSK and $2 M$-APSK as signal sets matched to the dihedral groups have been discussed considering all possible matched labelings. Automorphic Euclidean distance equivalence, rotational phase invariance, and Euclidean distance properties have been discussed. Given a pair of component codes, the best labeling among a subset of possible labelings has been identified only for specific ranges of values of the ratio of the Hamming distances of the component codes. One possible direction for further research is to find the best labeling for a given set of component codes at least for selected ranges of values for the ratio of the Hamming distances of the component codes.

Another direction to pursue is the comparison of performance with group codes over cyclic groups. Symmetric PSK signal sets can be labeled with cyclic group or with dihedral groups of the same order. It will be interesting to see under which labeling MSED is larger for identical code parameters like rate and dimension.

The signal space codes discussed in this correspondence admit minimal trellis [23], [24] and hence trellis-decoding techniques [25] can be used to decode. The method of construction of minimal trellis is first obtaining minimal trellises for the component codes and then taking their product. For the binary-code Kschischang-Sorokine algorithm [26] gives the minimal trellis and for the other code an algorithm to construct minimal trellis is given in [27].

\section{APPENDIX I}

\section{PROOF OF THEOREM 3}

For $d_{s}>d_{r}$, (17) becomes

$$
d_{m L} \geq \min \left\{\delta_{r} d_{r}, \delta_{0} d_{s}\right\} .
$$

i) Using $\delta_{0} d_{s}<\delta_{r} d_{r}$, (28) becomes

$$
d_{m L} \geq \delta_{0} d_{s}
$$

and from (17a) and Remark 2

$$
d_{S L}=\delta_{0} d_{s} .
$$

Therefore, $d_{m L} \geq d_{S L}$. Moreover, if we consider signal points corresponding to only all-s codewords then $d_{m L}>d_{S L}$, and if we consider signal points corresponding to only all- $r$ power codewords then also $d_{m L}>d_{S L}$, since $\delta_{r} d_{r}>\delta_{0} d_{s}$. Then, if equality is to hold in (28a) then any codeword that achieves equality is necessarily of the form

$$
\left(e, \cdots, e, z_{1}, e, \cdots, e, z_{d_{s}}, e \cdots e\right)
$$

where

$$
z_{k}=r^{M-1-m} s \text { or } r^{M-m} s, \quad k=0,1, \cdots, d_{s}
$$

for only $r^{M-1-m} s$ and $r^{M-m} s$ are points with Euclidean weight $\delta_{0}$.

If a minimum weight vector of $C_{s}$ is present also in $C_{r}$ then the MSED is $\delta_{0} d_{s}$ for all labelings since this Euclidean weight will be achieved by either the minimum-weight vector or a scalar multiple of it depending on $m L$.

ii) Using $\delta_{0} d_{s}>\delta_{r} d_{r}$, (17) becomes

$$
d_{m L} \geq \delta_{r} d_{r}
$$

and for SL, we always have

$$
\delta_{0} d_{s} \geq d_{S L}
$$

Case a) Restricting the consideration to within the class of all- $s$ codewords $d_{m L}=\delta_{m} d_{s}>\delta_{0} d_{s} \geq d_{S L}$.

Case b) Restricting the consideration to within the class of all $r$-power codewords, $d_{m L}=d_{S L}$.

Case c) Restricted to within the class of vectors not covered in cases a) and b) above, we have

$$
d_{m L} \geq \delta_{0} \max \left\{d_{r}, d_{s}\right\}=\delta_{0} d_{s} \geq d_{S L} .
$$

iii) Using $\delta_{0} d_{s}>\lambda d_{r}$ and $\delta_{r}<\lambda$, (28) gives $d_{m L} \geq \delta_{r} d_{r}$.

In this case, any vector that is not all $r$-power codeword will have minimum distance at least $\delta_{0} d_{s}$ which is larger than $\lambda d_{r}$. A minimum Hamming weight vector in $C_{r}$ can at most have Euclidean weight $\lambda d_{r}$. Hence the minimum Euclidean weight is achieved by an all- $r$ power vector for any labeling. Hence the $d_{m L}$ is independent of $m$, including $M D L$ and $S L$.

Q.E.D.

\section{APPENDIX II \\ PROOF OF THEOREM 5}

For $d_{s}<d_{r}$, (16) becomes

$$
d_{m L_{\phi}} \geq \min \left\{\delta_{\phi} d_{r}, \delta_{m_{\phi}} d_{s}\right\} .
$$

i) Let the angle of symmetry introduced to $m L$ be $\phi>0$, where $d_{s} / d_{r} \leq \delta_{\phi} / \delta_{\boldsymbol{m}_{\phi}}<\delta_{0} / \delta_{\boldsymbol{m}}$. We have

$$
d_{s} / d_{r}<\delta_{\phi} / \delta_{\boldsymbol{m}_{\phi}}
$$

Using (31) in (30) and from Remark 2, we have

$$
d_{\boldsymbol{m} L_{\phi}}=\delta_{\boldsymbol{m}_{\phi}} d_{s} .
$$

As $\phi$ increases, the right-hand side of (31) decreases and that of (32) increases. Moreover, (31) and (32) together imply that up to the maximum value of $\phi$ satisfying (31), the MSED will increase unless $M$ is odd and $\boldsymbol{m}=\frac{M-1}{2}$, in which case the MSED will decrease. Substituting the values of $\delta_{\phi}$ and $\delta_{\boldsymbol{m}_{\phi}}$ in (33), and after rearranging terms, we get (21). If $\phi<0$, then the right-hand side of (31) increases and the right-hand side of (34) decreases.

ii) Let $\phi$ be such that $\delta_{\phi} / \delta_{m_{\phi}}<d_{s} / d_{r}<1$. Then, using (30), we have $d_{m L_{\phi}}>\delta_{\phi} d_{r}$. For $\delta<0$, from (22b), we have

$$
d_{S L_{\phi}} \geq \min \left\{\delta_{\phi} d_{r}, \delta_{\phi} d_{s}\right\}=\delta_{\phi} d_{s} .
$$

Now, since $d_{r}>d_{s}$, we have $d_{m L_{\phi}}>d_{S L_{\phi}}$. Substituting the values for $\delta_{\phi}$ and $\delta_{m_{\phi}}$ we get the first inequality of (22a).

For $0<\phi<\pi / M$, we have

$$
d_{S L_{\phi}} \geq \min \left\{\delta_{\phi} d_{r}, \delta_{0_{\phi}} d_{s}\right\}=\delta_{\phi} d_{s}
$$

and from Remark $1, \delta_{0_{\phi}}>\delta_{\phi}$ and hence $d_{m L_{\phi}}>d_{S L_{\phi}}$.

\section{APPENDIX III}

Proof of THEOREM 7

Using $d_{s}>d_{r}$, (16) becomes

$$
d_{m L_{\phi}} \geq \min \left\{\delta_{r} d_{r}, \delta_{\phi} d_{s}\right\} .
$$

i) Since $\delta_{\phi} \leq \delta_{0}$, we have $d_{s} / d_{r}<\delta_{r} / \delta_{0}<\delta_{r} / \delta_{\phi}$, which when used in (33) gives

$$
d_{m L_{\phi}} \geq \delta_{\phi} d_{s}
$$

But from (16d)

$$
d_{S L_{\phi}}=\delta_{\phi} d_{s} .
$$

Therefore, $d_{m L_{\phi}} \geq d_{S L_{\phi}}$. Moreover, if we consider only all-s codewords then $d_{m L_{\phi}}>d_{S L_{\phi}}$, and if we consider only all-r power 
codewords then also $d_{m L_{\phi}}>d_{S L_{\phi}}$, since $\delta_{r} d_{r}>\delta_{\phi} d_{s}$. Hence, if equality is to hold in (34) then the codeword that achieves equality in (34) has to be necessarily of the form

$$
\left(e, \cdots, e, z_{1}, e, \cdots, e, z_{2}, e, \cdots, e, z_{d_{s}}, e \cdots e\right)
$$

where $z_{k}=r^{M-1-m} s, k=0,1, \cdots, d_{s}$, if $\phi>0$, and $z_{k}=r^{M-m} s$, $k=0,1, \cdots, d_{s}$, if $\phi<0$, for $r^{M-1-m} s$, and $r^{M-m} s$ being the only closest point with Euclidean weight $\delta_{\phi}$, respectively, when $\phi>0$ and $\phi<0$.

ii) Suppose for some $\phi$, we have

$$
\delta_{r} / \delta_{\phi}<d_{s} / d_{r}<4 / \delta_{\phi}
$$

Then (33) gives

$$
d_{m L_{\phi}} \geq \delta_{r} d_{r}
$$

and for $S L_{\phi}$, we always have

$$
\delta_{\phi} d_{s} \geq d_{S L_{\phi}} .
$$

Case a) Restricting the consideration to within the class of all-s codewords

$$
d_{m L_{\phi}}=\delta_{m_{\phi}} d_{s}>\delta_{\phi} d_{s} \geq d_{S L_{\phi}} .
$$

Case b) Restricting the considering to within the class of all $r$-power codewords, $d_{m L_{\phi}}=d_{S L_{\phi}}$.

Case c) Restricted to within the class of vectors not covered in a) and b),

$$
d_{m L_{\phi}} \geq \delta_{\phi} \max \left\{d_{r}, d_{s}\right\}=\delta_{\phi} d_{s} \geq d_{S L_{\phi}} .
$$

Cases a)-c) together imply $d_{m L_{\phi}} \geq d_{S L_{\phi}}$. With straightforward adjustments after substituting values for $\delta_{\phi}$ in (35), it is easy to check that $\phi$ has to be in the range given by (24) for (35) to hold.

iii) For all values of $\phi$ satisfying (25), we have $d_{s} / d_{r}>4 / \delta_{\phi}$, which when used in (33) gives $d_{m L_{\omega}} \geq \delta_{r} d_{r}$, since $4 d_{r}>\delta_{r} d_{r}$. In this case, any vector which consists of other than $r$-power terms will have the minimum distance at least $\delta_{\phi} d_{s}$ which is larger than $4 d_{r}$. A minimum Hamming weight vector in $C_{r}$ can at most have Euclidean weight $4 d_{r}$. Hence the minimum Euclidean weight is achieved by an all- $r$-power vector for any labeling. Hence the $d_{m L_{j}}$ is independent of $m$.

Q.E.D.

\section{ACKNOWLEDGMENT}

The authors wish to thank E. Biglieri, H. A. Loeliger, F. R. Kschischang, and R. Garello for sending the preprints of their work and the anonymous reviewers whose suggestions greatly improved the clarity of presentation.

\section{REFERENCES}

[1] E. Biglieri and C. Caire, "Symmetry properties of multilevel coded modulation," IEEE Trans. Inform. Theory, vol. 40, pp. 1630-1632, Sept. 1994.

[2] H. Imai and S. Hirakawa, "A new multilevel coding method using error correcting codes," IEEE Trans. Inform. Theory, vol. IT-23, pp. 371-377, May 1977.

[3] T. Kasami, T. Takata, T. Fujiwara, and S. Lin, "On linear structure and phase rotation invariant properties of block $M$-PSK modulation codes," IEEE Trans. Inform. Theory, vol. 37, pp. 164-167, Jan. 1991.

[4] _ _ "On multilevel block modulation codes," IEEE Trans. Inform. Theory, vol. 37, pp. 965-975, July 1991.

[5] S. I. Sayegh, "A class of optimum block codes in signal space," IEEE Trans. Commun., vol. COM-34, pp. 1043-1045, Oct. 1986.

[6] V. V. Ginzburg, "Multidimensional signals for a continuous channel," Probl. Inform. Transm., vol. 20, no. 1, pp. 20-34, Jan.-Mar. 1984.
[7] R. Garello and S. Benedetto, "Multilevel construction of block and trellis group codes," IEEE Trans. Inform. Theory, vol. 41, pp. 1257-1264, Sept. 1995.

[8] G. J. Pottie and D. P. Taylor, "Multilevel codes based on partitioning," IEEE Trans. Inform. Theory, vol. 35, pp. 87-98, Jan. 1989.

[9] E. L. Cusack, "Error control coding for QAM signalling," Electron. Lett., vol. 20, no. 2., pp. 62-63, Jan. 1984.

[10] C. Chen, T. Chen, and H. A. Loeliger, "Construction of linear ring codes for 6-PSK," IEEE Trans. Inform. Theory, vol. 40, pp. 563-566, Mar. 1994.

[11] F. R. Kschischang, P. G. deBuda, and S. Pasupathy, "Block coset codes for $M$-ary phase shift keying," IEEE J. Select. Areas Commun., vol. 7 , pp. 900-913, Aug. 1989.

[12] A. R. Calderbank and N. Seshadri, "Multilevel codes for unequal error protection," IEEE Trans. Inform. Theory, vol. 39, pp. 1234-1248, July 1993.

[13] R. H. Morelos Zaragoza and S. Lin, "QPSK block-modulation codes for unequal error protection," IEEE Trans. Inform. Theory, vol. 41, pp. 576-581, Mar. 1995.

[14] A. R. Calderbank, "Multilevel codes and multistage decoding," IEEE Trans. Commun., vol. 37, pp. 222-230, Mar. 1989.

[15] Y. Kofman, E. Zehavi, and S. Shamai, "Performance analysis of a multilevel coded modulation system," IEEE Trans. Commun., vol. 42, pp. 299-312, Feb./Mar./Apr. 1994.

[16] T. Takata, S. Ujita, T. Kasami, and S. Lin, "Multistage decoding of multilevel block $M$-PSK modulation codes and its performance analysis," IEEE Trans. Inform. Theory, vol. 39, pp. 1204-1218, July 1993.

[17] H. A. Loeliger, "On Euclidean space group codes," Ph.D. dissertation, Swiss Federal Inst. Technol., Zurich, Switzerland, 1992.

[18] _ "Signal sets matched to groups," IEEE Trans. Inform. Theory, vol. 37, pp. 1675-1682, Nov. 1991.

[19] G. D. Forney, Jr., "Geometrically uniform codes," IEEE Trans. Inform. Theory, vol. 37, pp. 1241-1260, Sept. 1991.

[20] C. Caire and E. Biglieri, "Linear block codes over cyclic groups," IEEE Trans. Inform. Theory, vol. 41, pp. 1246-1256, Sept. 1995.

[21] D. Slepian, "Group codes for the Gaussian channel," Bell Syst. Tech. J., vol. 47, pp. 575-602, Apr. 1968.

[22] A. A. Zain and B. Sundar Rajan, "Algebraic characterization of MDS group codes over cyclic groups," IEEE Trans. Inform. Theory, vol. 41, pp. 2052-2056, Nov. 1995.

[23] G. D. Forney, "Coset-codes-part I: Introduction and geometric classification," IEEE Trans. Inform. Theory, vol. 34, pp. 1123-1151, Sept. 1988.

[24] D. J. Muder, "Minimal trellises for block codes," IEEE Trans. Inform. Theory, vol. 34, pp. 1049-1053, Sept. 1988.

[25] J. K. Wolf, "Efficient maximum-likelihood decoding of linear block codes using a trellis," IEEE Trans. Inform. Theory, vol. IT-24, pp. 76-80, Jan. 1978.

[26] F. R. Kschischang and V. Sorokine, "On the trellis structure of block codes," IEEE Trans. Inform. Theory, vol. 41, pp. 1924-1937, Nov. 1995.

[27] V. V. Vazirani, H. Saran, and B. Sundar Rajan, "An efficient algorithm for constructing minimal trellises for codes over finite Abelian groups," IEEE Trans. Inform. Theory (Special Issue on Codes and Complexity), vol. 42, pp. 1839-1854, Nov. 1996. 